Pacific Journal of Mathematics

TRANSITIVE AND FULLY TRANSITIVE PRIMARY ABELIAN 


\section{TRANSITIVE AND FULLY TRANSITIVE PRIMARY ABELIAN GROUPS}

\section{PhILliP GRIFfith}

This paper is concerned with transitivity and full transitivity of primary abelian groups. It is well known that countable primary groups and primary groups without elements of infinite height are both transitive and fully transitive. The question of whether all primary groups are transitive or fully transitive was recently answered negatively by C. Megibben. Megibben's examples indicate that $p^{\omega} G$ may be transitive (fully transitive) while $G$ is not transitive (fully transitive). For $\beta$ an ordinal number, we investigate conditions on a primary group $G$ which will insure that $G$ is transitive (fully transitive) whenever $p^{\beta} G$ is transitive (fully transitive). Specifically, we show that if $G / p^{\beta} G$ is a direct sum of countable groups and $p^{\beta} G$ is fully transitive, then $G$ is fully transitive. The same result is established for transitivity except that $\beta$ is restricted to be a countable ordinal.

All groups considered in this paper are additively written primary abelian groups for a fixed prime $p$. For the most part, we follow the notation and terminology of [1]. All topological references will be to the $p$-adic topology. If $G$ is a $p$-primary group and if $\beta$ is an ordinal, we, define subgroups $G[p]$ and $p^{\beta} G$ as follows: $G[p]=\{x \in G \mid p x=0\}$; $p G=\{x \in G \mid x=p g, g \in G\}, p^{\beta} G=p\left(p^{\beta-1} G\right)$ if $\beta-1$ exists and

$$
p^{\beta} G=\bigcap_{\alpha<\beta} p^{\alpha} G
$$

if $\beta$ is a limit ordinal. If $x$ is an element of $G$ and $G$ is reduced we define the generalized height $h_{G}(x)$ and the generalized Ulm sequence $U_{G}(x)$ of $x$ by:

$$
\begin{aligned}
& h_{G}(x)= \begin{cases}\beta & \text { if } x \neq 0 \text { and } \beta+1 \text { is the first ordinal such that } \\
x \notin p^{\beta+1} G & \end{cases} \\
& U_{G}(x)=\left(\beta_{0}, \beta_{1}, \cdots, \beta_{i}, \cdots\right) \text { where } \beta_{i}=h_{G}\left(p^{i} x\right) \text { for each integer } i .
\end{aligned}
$$
The generalized Ulm sequences are partially ordered in the obvious term-by-term fashion, that is, $U_{G}(x) \geqq U_{G}(y)$ if and only if $h_{G}\left(p^{i} x\right) \geqq$ $h_{G}\left(p^{i} y\right)$ for all $i$. We assume, of course, that $\infty>\beta$ for all ordinals $\beta$.

Following Kaplansky [3], we call a reduced $p$-group $G$ fully transitive (transitive) if for each pair of elements $x$ and $y$ in $G$ with $U_{G}(x) \geqq U_{G}(y)\left(U_{G}(x)=U_{G}(y)\right)$ there exists an endomorphism (automorphism) $\varphi$ of $G$ such that $\varphi(y)=x$. Kaplansky has shown in [3] that 
reduced $p$-groups in which every pair of elements can be embedded in a countable direct summand are both transitive and fully transitive. In particular, countable reduced $p$-groups and $p$-groups without elements of infinite height (i.e. $p^{\omega} G=0$ ) are both transitive and fully transitive. Kaplansky suggested that all reduced $p$-groups might be such. However in [4], Meggibben exhibited a class of $p$-groups which are neither transitive nor fully transitive. A group $G$ in this class has the following properties:

(i) $p^{\omega} G$ is transitive.

(ii ) Not all endomorphisms (automorphisms) of $p^{\omega} G$ are induced by endomorphisms (automorphisms) of $G$.

Note that in order for (ii) to hold, $G$ must be uncountable. The above observations lead to the consideration of the following questions: Under a suitable hypothesis that ensures that endomorphisms (automorphisms) of $p^{\beta} G$ are induced by endomorphisms (automorphisms) of $G$, does it follow that $G$ must be fully transitive (transitive) whenever $p^{\beta} G$ is fully transitive (transitive)? In view of recent investigations by Nunke [5] and Hill and Megibben [2], the restriction we shall impose on $G$ is that $G / p^{\beta} G$ be a direct sum of countable groups. With this restriction on $G / p^{\beta} G$, affirmative answers are given in the present paper to both of the above questions-with the reservation that $\beta$ be a countable ordinal in the case of transitivity. Although the two concepts of transitivity and full transitivity might seem clearly related (there is some relationship, as is illustrated by Theorem 26 in [3]), the methods of proof are quite different. In dealing with the problem of full transitivity, the main result needed is one by Nunke [5]; whereas we rely on techniques of Hill and Megibben [2] for our result concerning transitivity.

2. Fully transitive primary groups. In what follows, the symbols $\sum$ and + will be used for direct sums; whereas the subgroup of a group $G$ generated by its subsets $S$ and $T$ will be denoted by $\{S, T\}$.

Lemma 2.1. Let $G$ be a p-primary group such that $G / p^{\beta} G$ is a direct sum of countable groups for some ordinal number $\beta$. Let $A$ be a finite subgroup of $G$ and $S=\left\{p^{\beta} G, A\right\}$. If $\theta$ is a homomorphism of $S$ into $G$ which does not decrease heights (computed in $G$ ), then $\theta$ extends to an endomorphism of $G$.

Proof. Consider the commutative diagram

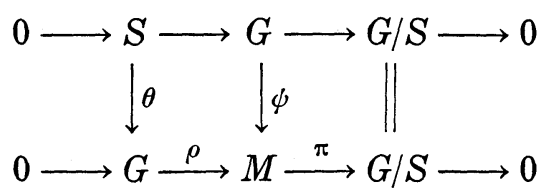


where $M=(G+G) / N, N=\{(-\theta(s), s) \mid s \in S\}$ and $\rho, \pi$ and $\psi$ are defined by $\rho: g \rightarrow(g, 0)+N, \pi:\left(g_{1}, g_{2}\right)+N \rightarrow g_{2}+S$ and $\psi: g \rightarrow(0, g)+N$. In the remainder of the section, we shall use the notations $G^{*}=\rho(G)$ and $\overline{\left(g_{1}, g_{2}\right)}$ for $\left(g_{1}, g_{2}\right)+N$ in $M$. To show that $\theta$ has the desired extension, it suffices to show that the bottom row of the above diagram splits, that is, it is enough to show that the exact sequence

$$
0 \longrightarrow G^{*} \longrightarrow M \longrightarrow M / G^{*} \longrightarrow 0 \text { splits. }
$$

We observe that $M / G^{*}$ is a direct sum of countable groups and that $p^{\beta}\left(M / G^{*}\right)=0$. This follows since $M / G^{*} \cong G / S, S=\left\{p^{\beta} G, A\right\}$ where $A$ is finite, and since $G / p^{\beta} G$ is a direct sum of countable groups such that $p^{\beta}\left(G / p^{\beta} G\right)=0$. Hence, $M / G^{*}$ is $p^{\beta}$-projective which implies $p^{\beta} \operatorname{Ext}\left(M / G^{*}, G^{*}\right)=0$. (For definitions of $p^{\beta}$-projective and $p^{\beta}$-purity see [6]). Thus, we need to show only that $0 \rightarrow G^{*} \rightarrow M \rightarrow M / G^{*} \rightarrow 0$ is $p^{\beta}$-pure exact. Since $p^{\beta}\left(M / G^{*}\right)=0$ and since $M / G^{*}$ is a direct sum of coutable groups, by applying Theorem 2.9 of [5], we can reduce the proof to exhibiting $p^{\alpha}\left(M / G^{*}\right)[p]=\left\{G^{*},\left(p^{\alpha} M\right)[p]\right\} / G^{*}$ for all $\alpha<\beta$. We shall need the following technical lemma.

LEMma 2.2. (The notation and setting is as above). If $\alpha<\beta$ and $x \in p^{\alpha}\left(M / G^{*}\right)$, then there is an element $w \in p^{\alpha} G$ such that $x=(\overline{0, w})+G^{*}$.

Proof. The proof is by induction on $\alpha$. For some $u \in G$, we have $x=(\overline{0, u})+G^{*}$. Suppose the assertain holds for all ordinals $\delta<\alpha$, $\alpha<\beta$, and that $x \in p^{\alpha}\left(M / G^{*}\right)$. If $\alpha-1$ is defined, $x=p y$ for some $y \in p^{\alpha-1}\left(M / G^{*}\right)$. By the induction hypothesis, $y=(\overline{0, v})+G^{*}$ where $v \in p^{\alpha-1} G$. Therefore, $\overline{(0, u)}+G^{*}=p(\overline{0, v})+G^{*}$ implies $(\overline{0, u})=$ $p \overline{(0, v)}+\overline{(g, 0)}$ where $\overline{(g, 0)} \in G^{*}$. Then $(0, u)=(0, p v)+(g, 0)+$ $(-\theta(s), s)$ for some $s \in S$. Now $s=b+a$ where $b \in p^{\beta} G$ and $a \in A$. Thus, we have $u=p v+s=p v+b+a$, which implies $u-a=p v+$ $b \in p^{\alpha} G$. Since $\left.a \in A,(\overline{0,-a})=(\overline{-\theta(\alpha}), 0\right) \in G^{*}$. Hence, setting $w=$ $u-a$, we obtain $w \in p^{\alpha} G$ and $x=(\overline{0, u})+G^{*}=(\overline{0, w})+G^{*}$ since $(\overline{0, w})=(\overline{0, u-a})+\overline{(-\theta(a), 0})$. If $\alpha$ is a limit ordinal, $p^{\alpha}\left(M / G^{*}\right)=$ $\bigcap_{\delta<\alpha} p^{\delta}\left(M / G^{*}\right)$. By the induction hypothesis, $\left.x=\overline{(0, u)}+G^{*}=\overline{\left(0, v_{\delta}\right.}\right)+G^{*}$ where $v_{\delta} \in p^{\delta} G$ for all $\delta<\alpha$. Hence, $(\overline{0, u})=\left(\overline{0, v_{\delta}}\right)+\left(\overline{g_{\delta}, 0}\right)$ where $\left(\overline{g_{\delta}, 0}\right) \in G^{*}$, for each $\delta$, yields $(0, u)=\left(0, v_{\delta}\right)+\left(g_{\delta}, 0\right)+\left(-\theta\left(s_{\delta}\right), s_{\delta}\right)$ where $s_{\delta} \in S$, for each $\delta<\alpha$. Now $s_{\delta}=b_{\delta}+a_{\delta}$ where $b_{\delta} \in p^{\delta} G$ and $a_{\delta} \in A$. Since $A$ is finite, there is a subset $\left[\delta_{\lambda}\right]_{\lambda \in A}$ of ordinals less than $\alpha$ such that $\sup \left[\delta_{\lambda} \mid \lambda \in \Lambda\right]=\alpha$ and $a_{\tilde{\delta}_{\lambda}}=\alpha$ for some $a \in A$ and all $\lambda \in \Lambda$. Hence, $u-a=v_{\delta_{\lambda}}+b_{\delta_{\lambda}} \in p^{\delta_{\lambda}} G$, for each $\lambda \in \Lambda$, implies $u-a \in \bigcap_{\lambda_{1}{ }_{1}} p^{\delta_{\lambda}} G$. But $\bigcap_{\lambda \in \Lambda} p^{\delta_{\Lambda}} G=\bigcap_{i<\alpha} p^{\delta} G=p^{\alpha} G$ since $\sup \left[\delta_{\lambda} \mid \lambda \in \Lambda\right]=\alpha$. Thus setting $w=u-a$, we again obtain $w \in p^{\alpha} G$ and $x=\left(\overline{0, w)}+G^{*}\right.$.

We now establish $p^{\alpha}\left(M / G^{*}\right)[p]=\left\{G^{*},\left(p^{\alpha} M\right)[p]\right\} / G^{*}$ for all $\alpha<\beta$. 
Suppose $x \in p^{\alpha}\left(M / G^{*}\right)[p]$. By Lemma 2.2, $\quad x=(\overline{0, w})+G^{*}$ where $w \in p^{\alpha} G$. Now $p(\overline{0, w}) \in G^{*}$ implies $p(\overline{0, w})=\overline{(g, 0)}$ for some $g \in G$, which yields $(0, p w)=(g, 0)+(-\theta(s), s)$ where $s \in S$. Therefore, $p w=s \in p^{\alpha+1} G$ and $\theta(s)=g \in p^{\alpha+1} G$ since $\theta$ does not decrease heights. Thus, $g=p h$ where $h \in p^{\alpha} G$. We have that $x=\left(\overline{0, w)}+G^{*}=\right.$ $(\overline{-h, w})+G^{*}$, where $(\overline{-h, w}) \in\left(p^{\alpha} M\right)[p]$ since $h, w \in p^{\alpha} G$ and since $p(\overline{-h, w)}=\overline{(-p h, p w)}=\overline{(-\theta(s), s)}=0 \in M$. Thus, we have shown that $p^{\alpha}\left(M / G^{*}\right)[p] \subseteq\left\{G^{*},\left(p^{\alpha} M\right)[p] / G^{*}\right\}$. The reverse inclusion is clear, thus the proof is complete.

We are now ready to prove the main result of this section.

THEOREM 2.3. Let $G$ be a p-primary group and let $\beta$ be an ordinal number. If $G / p^{\beta} G$ is a direct sum of countable groups and if $p^{\beta} G$ is fully transitive, then $G$ is fully transitive.

Proof. Let $x, y \in G$ such that $U_{G}(x) \leqq U_{G}(y)$ and let $p^{n}$ be the order of $x+p^{\beta} G$ in $G / p^{\beta} G$. Therefore, $p^{n} y \in p^{\beta} G$ since $U_{G}(x) \leqq U_{G}(y)$, and furthermore $U_{p \beta_{G}}\left(p^{n} x\right) \leqq U_{p \beta_{G}}\left(p^{n} y\right)$. Since $p^{\beta} G$ is fully transitive by hypothesis, there is an endomorphism $\varphi$ of $p^{\beta} G$ such that $\varphi\left(p^{n} x\right)=$ $p^{n} y$. Set $S=\left\{x, p^{\beta} G\right\}$ and define $\theta: S \rightarrow G$ by $\theta(m x+b)=m y+\varphi(b)$ where $b \in p^{\beta} G$ and $m$ is an integer. It is straight forward to check that $\theta$ is a homomorphism. To show that $\theta$ does not decrease heights (computed in $G$ ), it suffices to consider $m x+b \in S$ where $b \in p^{\beta} G$ and $m x \notin p^{\beta} G$. But $h_{G}(m x+b)=h_{G}(m x)$ since $b \in p^{\beta} G$ and since $m x \notin p^{\beta} G$. Therefore, $h_{G}(m x) \leqq \min \left[h_{G}(m y), h_{G}(\varphi(b))\right]$ implies that $h_{G}(m x+b) \leqq$ $h_{G}(\theta(m x+b))$. Thus $\theta$ does not decrease heights computed in $G$. Applying Lemma 2.1, we can extent $\theta$ to an endomorphism of $G$.

3. Transitive primary groups. In this section, our insistance that $\beta$ be a countable ordinal in Theorem 3.4 arises from the same restriction in Theorem 4 in [2]. Observe, however, that the only case left to be dealt with is that when $\beta$ is the first uncountable ordinal. Theorem 3.4 first requires the following lemmas.

Lemma 3.1. Let $A$ and $B$ be isomorphic countable p-groups and let $\beta$ be an ordinal. Suppose $a \in A$ and $b \in B$ such that $U_{A}(a)=U_{B}(b)$ and further suppose $p^{n}$ is the order of $a+p^{\beta} A$ in $A / p^{\beta} A$. If $\phi$ is an isomorphism of $p^{\beta} A$ onto $p^{\beta} B$ such that $\varphi\left(p^{n} a\right)=p^{n} b$, then $\phi$ extends to an isomorphism $\bar{\varphi}$ of $A$ onto $B$ such that $\bar{\varphi}(a)=b$.

The proof of this lemma is the same at that of Zippin's Theorem [7] which states: If $G$ is a countable reduced primary group, then every automorphism of $p^{\beta} G$ is induced by an automorphism of $G$. 
Lemma 3.2. Let $G$ be a p-group and let $\beta$ be an ordinal. If $C$ is a pure subgroup of $p^{\beta} G$ and if $H$ is a subgroup of $G$ such that $G / C=H / C+p^{\beta} G / C$, then $p^{\alpha} G \cap H=p^{\alpha} H$, for all $\alpha<\beta$.

Proof. The proof is by induction on $\alpha$. Suppose the assertain holds for all $\delta<\alpha$, where $\alpha \leqq \beta$. If $\alpha$ is a limit ordinal, then $p^{\alpha} H=\bigcap_{\delta<\alpha} p^{\delta} H=\bigcap_{\delta<\alpha}\left(p^{\delta} G \cap H\right)=\left(\bigcap_{\delta<\alpha} p^{\alpha} G\right) \cap H=p^{\alpha} G \cap H$. Therefore, we may assume $\alpha-1$ is defined. Suppose $x \in p^{\alpha-1} G$ such that $p x=h_{0} \in H$. Since $G=\left\{H, p^{\beta} G\right\}, x=h+y$ where $h \in H$ and $y \in p^{\beta} G$. It follows that $h=x-y \in p^{\alpha-1} G \cap H=p^{\alpha-1} H$ by the induction hypothesis. Since $p y=h_{0}-p h \in H \cap p^{\beta} G=C$, then $p y=p c$, for some $c \in C$ where $c \in p^{\beta} G \cap H \subseteq p^{\alpha-1} G \cap H=p^{\alpha-1} H$. Thus, $p x=p(h+c)$ and $h+c$ $\in p^{\alpha-1} H$. Hence, $p^{\alpha} G \cap H=p^{\alpha} H$.

LEMma 3.3. Let $G$ be a p-group such that $G / p^{\beta} G$ is a direct sum of countable groups and let $\beta$ be a countable ordinal. Suppose $p^{\beta} G$ is transitive and further suppose there is an automorphism $\theta$ of $G$ such that $\theta(a)=b$ whenever $a$ and $b$ satisfy:

(i) $U_{G}(a)=U_{G}(b)$

(ii) $p^{n} a=p^{n} b$ where $p^{n}$ is the order of $a+p^{\beta} G$ in $G / p^{\beta} G$. Then $G$ is transitive.

Proof. Let $a, b \in G$ such that $U_{G}(a)=U_{G}(b)$ and let $p^{n}$ be the order of $a+p^{\beta} G$ in $G / p^{\beta} G$. Since $U_{G}(a)=U_{G}(b)$, then $p^{n} b \in p^{\beta} G$ and $U_{p \beta_{G}}\left(p^{n} a\right)=U_{p \beta_{G}}\left(p^{n} b\right)$. Applying the transitivity of $p^{\beta} G$, there is an automorphism $\sigma$ of $p^{\beta} G$ such that $\sigma\left(p^{n} a\right)=p^{n} b$. Since $G / p^{\beta} G$ is a direct sum of countable groups and since $\beta$ is a countable ordinal, $\sigma$ extends to $\bar{\sigma}$ an automorphism of $G$, by Theorem 5 [2]. Set $a_{1}=\bar{\sigma}(a)$. Therefore, $p^{n} a_{1}=p^{n} b$ and $U_{G}\left(a_{1}\right)=U_{G}(a)=U_{G}(b)$ since $\bar{\sigma}$ is an automorphism of $G$. Thus, $a_{1}$ and $b$ satisfy (i) and (ii) of the hypothesis, and hence there is an automorphism $\theta$ such that $\theta\left(a_{1}\right)=b$. Setting $\psi=\theta \bar{\sigma}$, we obtain an automorphism of $G$ such that $\psi(a)=b$.

We now state the main theorem of this section.

THEOREM 3.4. Let $G$ be a p-primary group and let $\beta$ be a countable ordinal. If $G / p^{\beta} G$ is a direct sum of countable groups and if $p^{\beta} G$ is transitive, then $G$ is transitive.

Proof. Let $a, b \in G$ such that $U_{G}(a)=U_{G}(b)$ and let $p^{n}$ be the order of $a+p^{\beta} G$ in $G / p^{\beta} G$. By Lemma 3.3 we may assume $p^{n} a=p^{n} b$. There is a pure dense subgroup $C$ of $p^{\beta} G$ such that $p^{n} a=p^{n} b \in C$ and such that $C$ is a direct sum of countable groups. Choose subgroups $H$ and $K$ of $G$ such that $a \in H, b \in K$ and such that 


$$
G / C=H / C+p^{\beta} G / C=K / C+p^{\beta} G / C .
$$

By Lemma 3.2, $p^{\beta} H=p^{\beta} G \cap H=C=p^{\beta} G \cap K=p^{\beta} K$. Since $C$ is a direct sum of countable groups, $p^{\beta} H=p^{\beta} K=C, H / C \cong G / p^{\beta} G \cong K / C$, and since $\beta$ is a countable ordinal, it follows from Theorem 4 [2] that the identity automorphism on $C$ may be extended to an isomorphism $\sigma$ of $H$ onto $K$. By Proposition 2.13 [5], $H$ and $K$ are both direct sums of countable groups. Using the isomorphism $\sigma$, we can find decompositions of $H$ and $K$, respectively, which satisfy:

(i) $H=A+S$ and $K=B+T ; a \in A$ and $b \in B$.

(ii) $A \cong B$ and $S \cong T$ under the isomorphism $\sigma$.

(iii) $p^{\beta} A=p^{\beta} B$ and $p^{\beta} S=p^{\beta} T$.

(iv) $|A|=|B| \leqq \boldsymbol{\aleph}_{0}$.

Since $p^{\alpha} G \cap H=p^{\alpha} H$ and $p^{\alpha} G \cap K=p^{\alpha} K$ for all $\alpha \leqq \beta$ by Lemma 2.3, since $p^{n} a=p^{n} b$ where $p^{n}$ is the order of $a+p^{\beta} G$ and $b+p^{\beta} G$ in $G / p^{\beta} G$, and since $U_{G}(a)=U_{G}(b)$, it follows that $U_{H}(a)=U_{K}(b)$. Hence, $U_{A}(a)=U_{B}(b)$. Thus, letting $\phi$ be the identity automorphism on $p^{\beta} A=p^{\beta} B$ and applying Lemma 3.1, we obtain an isomorphism $\bar{\varphi}$ of $A$ onto $B$ such that $\bar{\varphi}(a)=b$ and such that $\bar{\varphi}$ extends $\varphi$. Since $S$ and $T$ are isomorphic direct sums of countable groups with $p^{\beta} S=p^{\beta} T$ and since $\beta$ is a countable ordinal, by Theorem 4 [2] there is an isomorphism $\psi$ of $S$ onto $T$ such that $\psi$ is the identity on $p^{\beta} S=p^{\beta} T$. Hence, by defining $\theta(a+s)=\bar{\varphi}(a)+\psi(s)$ where $a \in A$ and $s \in S$, we establish an isomorphism $\theta$ of $H$ onto $K$ such that $\theta$ extends the identity automorphism on $p^{\beta} H=p^{\beta} K=C$ and such that $\theta(a)=b$. The proof is completed by extending $\theta$ to an automorphism $\bar{\theta}$ of $G$ by defining $\bar{\theta}(h+x)=$ $\theta(h)+x$ for $h \in H$ and $x \in p^{\beta} G$.

\section{REFERENCES}

1. L. Fuchs, Abelian Groups, Publ. House Hungar. Acad. Sci., Budapest, 1958.

2. Paul Hill and Charles Megibben, Extending automorphisms and lifting decompositions in abelian groups, Math. Ann. (to appear)

3. I. Kaplansky, In finite Abelian Groups, University of Michigan Press, Ann Arbor, 1954.

4. Charles Megibben, Large subgroup and small homomorphism, Michigan Math. J. 13 (1966), 153-160.

5. R. J. Nunke, Homology and direct sums of countable abelian groups, (to appear).

6. - "Purity and Subfunctors of the Identity", Topics in Abelian Groups, Scott, Foresman and Company, Chicago, Illinois, 1963.

7. Z. Zippin, Countable torsion groups, Ann. of Math. 36 (1935), 86-99.

Received January 26, 1967. The author wishes to acknowledge support by the National Aeronautics and Space Administration Grant NsG(T)-52. 


\section{PACIFIC JOURNAL OF MATHEMATICS}

\section{EDITORS}

\section{H. ROYDEN}

Stanford University

Stanford, California
J. DugundJI

Department of Mathematics

Rice University

Houston, Texas 77001

RICHARD ARENS

University of California

Los Angeles, California 90024

Seattle, Washington 98105

\section{ASSOCIATE EDITORS}
E. F. BeCKENBACH
B. H. NeumanN
F. WOLF
K. YOSIDA

\section{SUPPORTING INSTITUTIONS}

\author{
UNIVERSITY OF BRITISH COLUMBIA \\ CALIFORNIA INSTITUTE OF TECHNOLOGY \\ UNIVERSITY OF CALIFORNIA \\ MONTANA STATE UNIVERSITY \\ UNIVERSITY OF NEVADA \\ NEW MEXICO STATE UNIVERSITY \\ OREGON STATE UNIVERSITY \\ UNIVERSITY OF OREGON \\ OSAKA UNIVERSITY \\ UNIVERSITY OF SOUTHERN CALIFORNIA
}

\author{
STANFORD UNIVERSITY \\ UNIVERSITY OF TOKYO \\ UNIVERSITY OF UTAH \\ WASHINGTON STATE UNIVERSITY \\ UNIVERSITY OF WASHINGTON \\ * * * * \\ AMERICAN MATHEMATICAL SOCIETY \\ CHEVRON RESEARCH CORPORATION \\ TRW SYSTEMS \\ NAVAL WEAPONS CENTER
}

Mathematical papers intended for publication in the Pacific Journal of Mathematics should be in typed form or offset-reproduced, double spaced with large margins. Underline Greek letters in red, German in green, and script in blue. The first paragraph or two must be capable of being used separately as a synopsis of the entire paper. It should not contain references to the bibliography. Manuscripts, in duplicate if possible, may be sent to any one of the four editors. All other communications to the editors should be addressed to the managing editor, Richard Arens, University of California, Los Angeles, California 90024.

Each author of each article receives 50 reprints free of charge; additional copies may be obtained at cost in multiples of 50 .

The Pacific Journal of Mathematics is published monthly. Effective with Volume 16 the price per volume (3 numbers) is $\$ 8.00$; single issues, $\$ 3.00$. Special price for current issues to individual faculty members of supporting institutions and to individual members of the American Mathematical Society: $\$ 4.00$ per volume; single issues $\$ 1.50$. Back numbers are available.

Subscriptions, orders for back numbers, and changes of address should be sent to Pacific Journal of Mathematics, 103 Highland Boulevard, Berkeley 8, California.

Printed at Kokusai Bunken Insatsusha (International Academic Printing Co., Ltd.), 7-17, Fujimi 2-chome, Chiyoda-ku, Tokyo, Japan.

PUBLISHED BY PACIFIC JOURNAL OF MATHEMATICS, A NON-PROFIT CORPORATION

The Supporting Institutions listed above contribute to the cost of publication of this Journal, but they are not owners of publishers and have no responsibility for its content or policies. 


\section{Pacific Journal of Mathematics \\ Vol. 25, No. $2 \quad$ October, 1968}

Martin Aigner, On the tetrahedral graph ..................... 219

Gregory Frank Bachelis, Homomorphisms of annihilator Banach

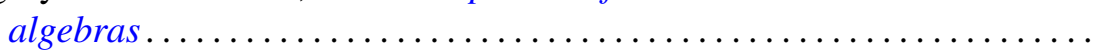

Phillip Alan Griffith, Transitive and fully transitive primary abelian groups.......................................... 249

Benjamin Rigler Halpern, Fixed points for iterates . . . . . . . . . . . . . 255

James Edgar Keesling, Mappings and dimension in general metric spaces ......................................... 277

$\mathrm{Al}$ (Allen Frederick) Kelley, Jr., Invariance for linear systems of ordinary differential equations ................................ 289

Hayri Korezlioglu, Reproducing kernels in separable Hilbert spaces . . . . . 305

Gerson Louis Levin and Wolmer Vasconcelos, Homological dimensions and Macaulay rings ................................. 315

Leo Sario and Mitsuru Nakai, Point norms in the construction of harmonic

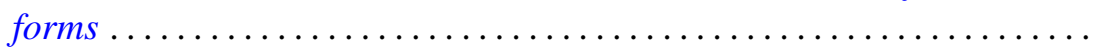

Barbara Osofsky, Noncommutative rings whose cyclic modules have cyclic injective hulls ..................................... 331

Newton Tenney Peck, Extreme points and dimension theory........... 341

Jack Segal, Quasi dimension type. II. Types in 1-dimensional spaces ...... 353

Michael Schilder, Expected values of functionals with respect to the Ito distribution ...

Grigorios Tsagas, A Riemannian space with strictly positive sectional curvature

John Alexander Williamson, Random walks and Riesz kernels . . 